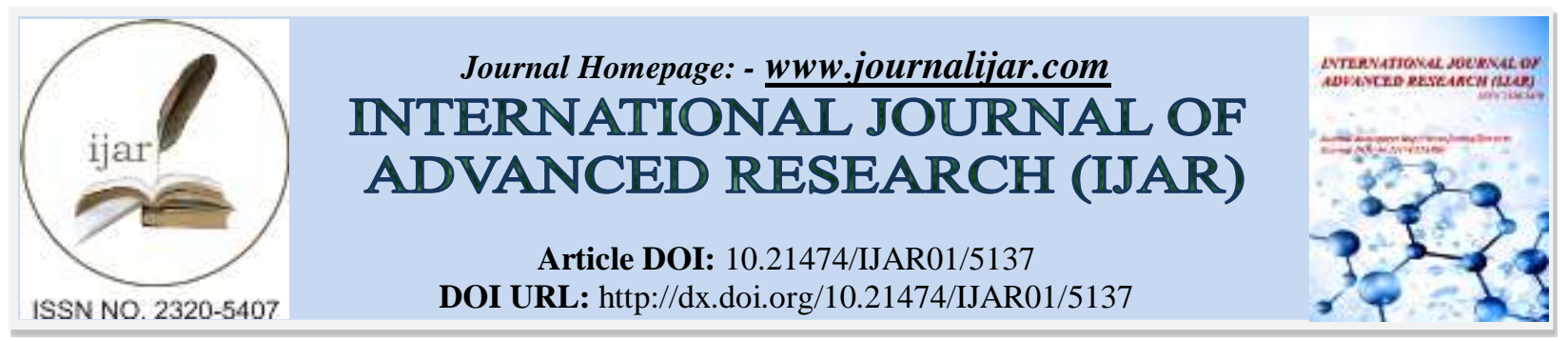

RESEARCH ARTICLE

\title{
ASSOCIATION OF ASYMPTOMATIC NEUROENDOCRINE TUMOUR WITH RAPID-ONSET OBESITY, HYPOVENTILATION, HYPOTHALAMIC DYSFUNCTION, AND AUTONOMIC DYSFUNCTION (ROHHAD) SYNDROME.
}

Asmaa Milyani ${ }^{1}$, Abdulmoein Eid Al-Agha ${ }^{2}$, and Mashael Alzanbagi ${ }^{3}$.

1. MBBS, Student at Faculty of Medicine at King Abdul Aziz University, Jeddah, Saudi Arabia, P. O. BOX. 80215.

2. Professor of Paediatric Endocrinology, King Abdulaziz University Hospital, Paediatric Department, P. O. BOX 80215, Jeddah 21589, Saudi Arabia.

3. MBBS, Student at Faculty of Medicine at King Abdul Aziz University, Jeddah, Saudi Arabia, P. O. BOX. 80215 .

\section{Manuscript Info}

Manuscript History

Received: 11 June 2017

Final Accepted: 13 July 2017

Published: August 2017

Key words:-

Obesity, Hypothalamic, Autonomic,

Dysfunction, Neuroendocrine, Tumour.

\begin{abstract}
Acknowledgment: We thank Dr. Mashael Saber for her excellent assistance and support in accomplishing this work.

Disclosure: Authors have no conflict of interests, and the work was not supported or funded by any drug company.

Word count: 1,183 words

Number of tables: 2

Number of figures: 3

Consent for publication: Consent was obtained from the patients' legal guardian for this publication.

Ethical approval and consent to participate: None applicable.

Abstract: The rapid onset of obesity in the setting of hypoventilation, hypothalamic dysfunction and autonomic dysfunction constitutes the syndrome of ROHHAD-NET, with some cases presenting with a neuroendocrine tumour. It is a rare fatal disease due to respiratory failure that necessitates the early recognition and consequent provision of ventilatory support to improve outcome.
\end{abstract}

Copy Right, IJAR, 2017,. All rights reserved.

\section{Introduction:-}

The constellation of symptoms involving rapid-onset obesity, hypoventilation, hypothalamic dysfunction, autonomic dysfunction with or without the association of neuroendocrine tumours comprises the rare syndrome of ROHHADNET. ROHHAD is a rare disorder with less than a 100 cases up to date reported worldwide (1). Unfortunately, mortality rate is high with cardiopulmonary arrest topping as the most common cause of death. However, early ventilatory support proposes a possible improvement in the entailing poor prognosis.

Onset is usually presumed to be at ages between 2 and 4 years with hyperphagia and dramatic weight gain appearing as the most consistent presentation. They are reported to be healthy prior to these symptoms (2-4). Behavioral problems, psychotic disorders, mood disorders, and personality changes were also reported in relation to patients suffering from this syndrome (5). 
The underlying aetiology is yet to be identified, but it appears to be an immune mediated process. A subset of affected patients has a neural crest tumor that presumably precedes the onset of the clinical syndrome. In this subset, the syndrome may occur due to reactivity of the patient's immune system to the presence of the neural crest tumor (6).

We report the recognition of a second case of ROHHAD-NET syndrome in Saudi Arabia.

\section{Case Report:-}

A 4-year-old Afghani girl living in Saudi Arabia presented to King AbdulAziz University Hospital and was admitted as a case of rapid-onset weight gain and episodes of hypoventilation. Her problems began when she was at 3 years of age, and over the course of the subsequent 9 months, her parents took notice of the developing abnormalities: rapid weight gain $(21 \mathrm{~kg})$ accompanied by polyphagia, unexplained behavioral changes (aggression, seclusion), central cyanotic attacks secondary to hypoventilation, and an altered consciousness with increased drowsiness and hypersomnia.

Upon examination, she was vitally and haemodynamically stable, however her extremities were cold. Her anthropometric measures showed a height of $123 \mathrm{~cm}(+5.6 \mathrm{SD})$, weight of $36 \mathrm{~kg}(+12.13 \mathrm{SD})$, and a head circumference of $54 \mathrm{~cm}(+3.14 \mathrm{SD})$. There were no dysmorphic features and all her physical evaluations were unremarkable apart from breast development (Tanner stage 2) in absence of pubic hair. Pelvic and abdominal ultrasound studies confirmed normal reproductive anatomy. Full endocrine profile was requested and showed Hypothalamic-Pituitary Axis (HPA) disturbance in the form of elevated serum levels of Prolactin [table 1] and a positive GnRH stimulation test for central Precocious Puberty (PP) [table 2] without supporting radiological evidence [figure 1].

During her hospitalisation, she fell into severe respiratory distress with hypoventilation and oxygen desaturation. Her blood gas analysis showed marked hypercapnia and respiratory acidosis [table 1] and therefore was put on continuous BiPAP with significant improvement. She also developed hypertension and tachycardia. After she was stabilised, the use of assisted ventilation was limited to sleeping hours only as she continued to suffer from episodes of nocturnal hypoventilation.

Based on the above-mentioned clinical scenario and the corroborative sequence of events, the suspicion of ROHHAD-NET syndrome was in query and an abdominal computed tomography (CT) scan was ordered. A $6.8 \times 4 \times 1.8 \mathrm{~cm}$ homogenous, regular, well-defined retroperitoneal soft tissue mass was found at the aortic bifurcation [figure 2]. Nuclear medical scanning using MIBG scintigraphy [figure 3] found that the mass was compatible with a benign neuroendocrine tumour.

Two weeks later, the patient presented to our ER with hyponatraemic dehydration, generalised oedema, and oliguria. Biochemical investigations [table 1] suggested the provisional diagnosis of Syndrome of Inappropriate Antidiuretic Hormone secretion (SIADH), further highlighting the dysfunction of the HPA.

\section{Discussion:-}

We present a case of ROHHAD-NET syndrome due to complete fulfillment of the diagnostic criteria.

She was born to non-consanguineous parents at full term after an uneventful pregnancy without any reported perinatal complications. Consistent with the published literature, her medical conditions had only started when she was three years of age, when the rapid onset obesity took place concurrently with hyperphagia. Difficulties surrounding the diagnosis of ROHHAD in relation to formal diagnosis due to the frequency of hyperphagia as a cause of obesity were addressed. More common endocrine conditions that may have been implicated such as Cushing's disease and Hypothyroidism were ruled out in the presence of normal cortisol and thyroid profiles [table 1]. Syndromic causes of morbid obesity such as Prader-Willi and Bardet-Biedl were considered due to the possibility of hypothalamic dysfunction but there was no indication to pursue further examination in the absence of dysmorphic features, intellectual disability, failure to thrive, hypotonia, developmental delay, congenital anomalies and retinitis pigmentosa. Monogenic obesity syndromes comprising mutations within the leptin-melanocortin genes were excluded due to the lacking of the essentially diagnostic hypogonadrotropic-hypogonadism associated with congenital leptin deficiency and the red hair and hypocortisolism found in cases of leptin receptor proopiomelanocortin (POMC) deficiency. 
She also had suffered several episodes of severe respiratory distress necessitating intermittent assisted ventilation and complete oxygen dependency. Entertaining the association between alveolar hypoventilation and other cardiac and pulmonary disorders, heart and lung diseases were investigated. But in the verified setting of normal cardiopulmonary anatomy and functioning, the possibility of an underlying primary pathology for the respiratory failure has been eliminated.

Unlike the only other reported case of ROHHAD syndrome in Saudi Arabia (7), a neuroendocrine tumour (NET) had been identified in our patient, and following excision, was confirmed by histopathology to have been a ganglion neuroblastoma. It is noteworthy to mention that in this patient, the presence of her NET was not symptomatic, but because of previously reported cases (8), radiological investigations were done with positive association. Therefore, we raise the necessity to screen for neuroendocrine tumours in suspected cases.

Variables of HPA disturbance were witnessed in the form of an otherwise unexplained abnormality in thermoregulation varying from episodes of hyperthermia (39C) to hypothermia (29C), hyperprolactinaemia, central precocious puberty, and hyponatraemia associated with inappropriate ADH secretion. While other case reports had reported concurrent abnormal radiological results of brain anatomy $(1,8,9)$, in this case, all brain MRI and CT scans were unremarkable ruling out central nervous system pathology [figure 3].

Her cold peripheries and the development of intermittent episodes of hypertension in the absence of an underlying renal pathology support the presence of an autonomic dysfunction that is conventional in the considered diagnostic circumstances. There were also no hot flushes, diaphoresis, tachycardia, or abnormal blood glucose values that would raise the suspicion of catecholamine excess. Behavioral changes experienced by our patient were similar to those reported in Aljabban's et al (8), further supporting the notion of psychiatric involvement in ROHHAD syndrome. These symptoms were manifested in the form of mood alteration, rage attacks, and aggressive behavior, in addition to recurrent fatigue, social withdrawal, prolonged periods of sleep and difficulty staying awake during the day.

\section{Conclusion:-}

Although ROHHAD-NET syndrome is a rare condition that is unrecognised by many, it is established with enough reported cases and mortality to validate it as a considerable concern. Thereby, timely recognition of the presenting characteristics requires a high suspicion index for cases that present with an unexplained rapid-onset obesity. We aim to increase awareness of ROHHAD syndrome amongst general paediatricians and paediatric endocrinologists. In addition, we recommend that all patients suspected to have ROHHAD syndrome be screened for the presence of neuroendocrine tumours, even if they ere asymptomatic.

\begin{tabular}{|l|l|l|}
\hline & Result & Reference value \\
\hline Prolactin (mIU/L) & 1411 & $83-539$ \\
\hline TSH (uIU/L) & 1.34 & $0.27-4.2$ \\
\hline Free T4 (Pmol/L) & 17.1 & $12-22$ \\
\hline Cortisol (nmol/L) & 437.7 & $138-636$ \\
\hline IGF-1 (ng/ml) & 153 & $33-172$ \\
\hline IGFBP-3 (ng/ml) & 4950 & $1100-5200$ \\
\hline Glucose (mmol/L) & 5.8 & $3.9-6.7$ \\
\hline Albumin (g/L) & 37 & $40.2-47.6$ \\
\hline Alkaline Phosphatase (ALP) (U/L) & 191 & $156-369$ \\
\hline Aspartate Aminotransferase (AST) (U/L) & 14 & $15-37$ \\
\hline Alanine Transaminase (ALT) (U/L) & 22 & $12-78$ \\
\hline Creatinine (umol/L) & 28.1 & $53-115$ \\
\hline Blood Urea Nitrogen (mmol/L) & 4.7 & $2.5-6.4$ \\
\hline Serum sodium (mmol/L) & 118 & $136-145$ \\
\hline Urine sodium (mmol/L) & 73 & $40-220$ \\
\hline Chloride (mmol/L) $(\mathbf{m})$ & 84 & $98-107$ \\
\hline Potassium (mmol/L) & 4.5 & $3.5-5.1$ \\
\hline Serum osmolality (mOS/kg) & 243 & $285-295$ \\
\hline Urine osmolality (mOS/kg) & 518 & $38-1400$ \\
\hline
\end{tabular}




\begin{tabular}{|l|l|l|}
\hline Plasma pH & 7.24 & $7.35-7.45$ \\
\hline PCO2 $(\mathbf{k P a})$ & 13.8 & $4.7-6.0$ \\
\hline PO2 $(\mathbf{k P a})$ & 6.62 & $10.5-13.5$ \\
\hline Bicarbonate $(\mathbf{m E q} / \mathbf{L})$ & 23 & $22-26$ \\
\hline Base excess $(\mathbf{m l}$ litre) & +2 & -2 to +2 \\
\hline
\end{tabular}

Table 1:- Biochemical investigations

\begin{tabular}{|l|l|l|l|}
\hline & FSH (unit) & LH (unit) & Oestradiol (Pmol/L) \\
\hline Pre-dose & 7.31 & 1.88 & 74.22 \\
\hline $\mathbf{1 5}$ min & 13.25 & 9.52 & 65.68 \\
\hline $\mathbf{3 0}$ min & 14.49 & 10.11 & 68.68 \\
\hline $\mathbf{4 5}$ min & 15.11 & 10.44 & 83.51 \\
\hline $\mathbf{6 0}$ min & 15.55 & 10.56 & 83.66 \\
\hline $\mathbf{9 0}$ min & 19.15 & 12.95 & 83.43 \\
\hline $\mathbf{1 2 0}$ min & 25.77 & 18.34 & 83.63 \\
\hline
\end{tabular}

Table 2:- Positive GnRH stimulation test for Precocious Puberty (PP)

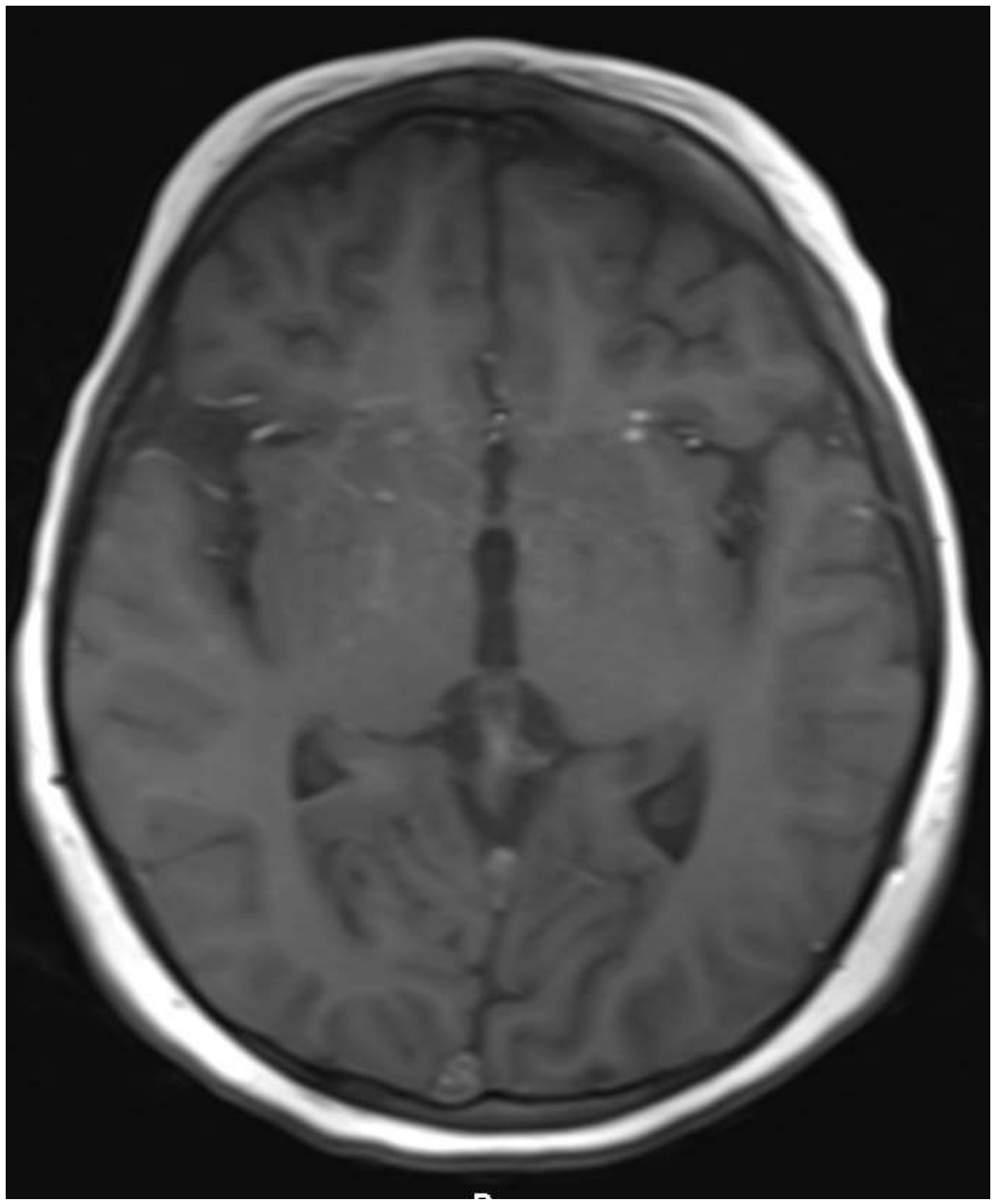

Figure 1:- Normal Brain MRI 


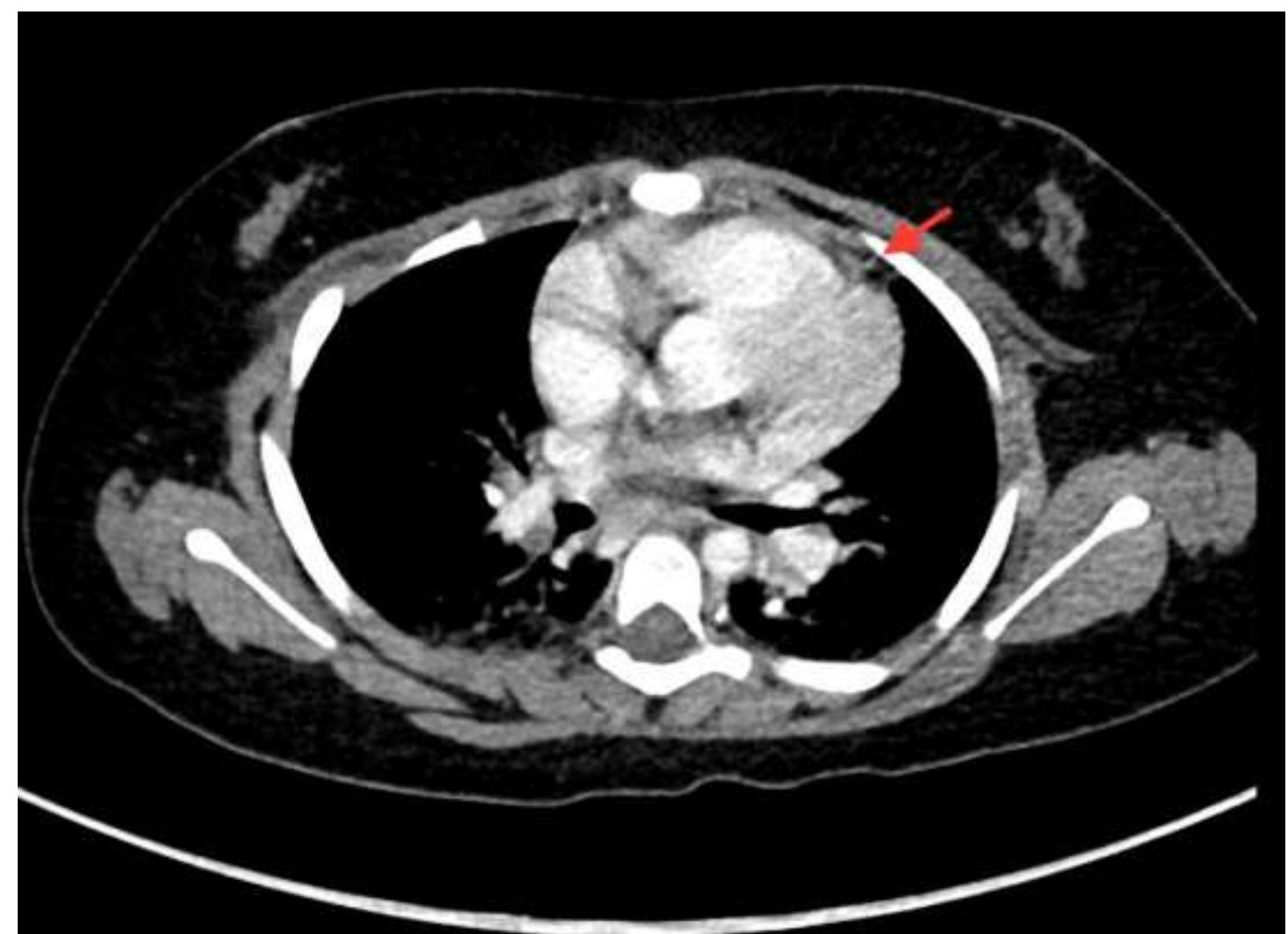

Figure 2:- Abdominal CT scan showing retroperitoneal mass, likely presenting a neurogenic tumour.

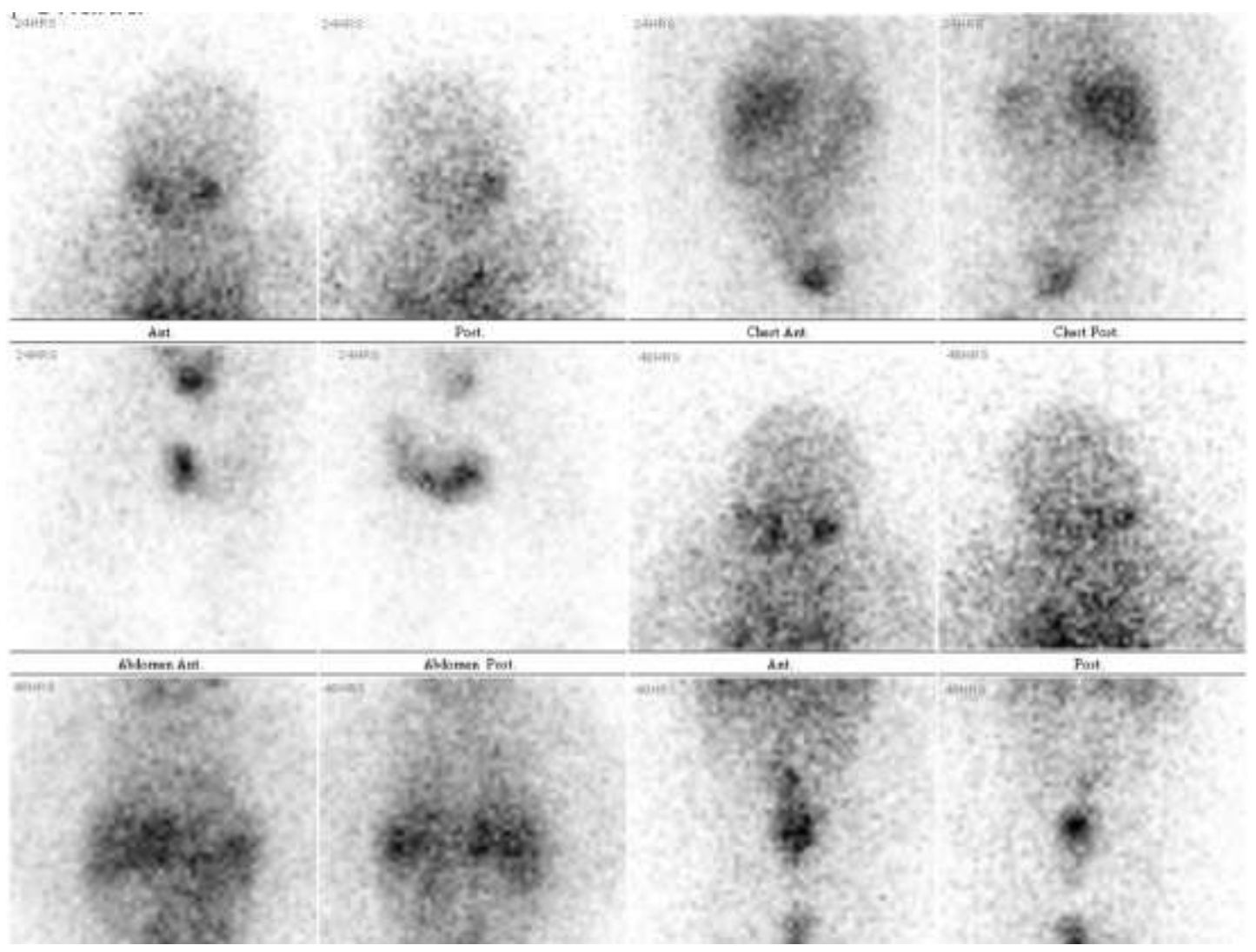

Figure 3:- Nuclear Imaging scan showing an abdominal mass compatible with a neuroendocrine tumour. 


\section{References:-}

1. Kocaay P, Zeynep Ş, Çamtosun E, Kendirli T, Berbero M. Case Report ROHHAD Syndrome: Reasons for Diagnostic Dif fi culties in Obesity. 2014;6(4):254-7.

2. Ize-Ludlow D, Gray JA, Sperling MA, Berry-Kravis EM, Milunsky JM, Farooqi IS, et al. Rapid-onset obesity with hypothalamic dysfunction, hypoventilation, and autonomic dysregulation presenting in childhood. Pediatrics [Internet]. 2007;120(1):e179-88. Available from: http://www.ncbi.nlm.nih.gov/pubmed/17606542\%5Cnhttp://pediatrics.aappublications.org.proxy.queensu.ca/co ntent/120/1/e179.long

3. De Pontual L, Trochet D, Caillat-Zucman S, Abou Shenab OA, Bougneres P, Crow Y, et al. Delineation of late onset hypoventilation associated with hypothalamic dysfunction syndrome. Pediatr Res. 2008;64(6):689-94.

4. Bougnères $\mathrm{P}$, Pantalone L, Linglart A, Rothenbühler A, Le Stunff $\mathrm{C}$. Endocrine manifestations of the rapidonset obesity with hypoventilation, hypothalamic, autonomic dysregulation, and neural tumor syndrome in childhood. J Clin Endocrinol Metab. 2008;93(10):3971-80.

5. Erensoy H, Ceylan M, Evrensel A. Psychiatric Symptoms in Rapid-onset Obesity with Hypothalamic Dysfunction, Hypoventilation, and Autonomic Dysregulation Syndrome and its Treatment: A Case Report. Chin Med J (Engl) [Internet]. 2016;129(2):242. Available from: http://www.cmj.org/text.asp?2016/129/2/242/173550

6. Malatack J, Editor A. Rapid Onset Obesity-A Case Report. 2016;2016.

7. Al-Harbi AS, Al-Shamrani A, Al-Shawwa BA. Rapid-onset obesity, hypothalamic dysfunction, hypoventilation, and autonomic dysregulation in Saudi Arabia. Saudi Med J. 2016;37(11):1258-60.

8. L. A, L. K, N.A. B, M.F. A. Rapid-onset obesity, hypoventilation, hypothalamic dysfunction, autonomic dysregulation and neuroendocrine tumor syndrome with a homogenous enlargement of the pituitary gland: a case report. J Med Case Rep [Internet]. 2016;10(1):1-9. Available from: http://www.jmedicalcasereports.com/articles/browse.asp\%0Ahttp://ovidsp.ovid.com/ovidweb.cgi?T=JS\&PAGE $=$ reference $\& D=e m e d 18 b \& N E W S=N \& A N=613329545$

9. Valea a. Morbid Child Obesity with Possible Rohhadnet-Rohhad Syndrom. Case Report. Acta Endocrinol [Internet]. 2014;10(3):501-10. Available from: http://www.acta-endo.ro/Archive/Abstract?doi=2014.501 